\title{
Study of fragmentation function at Belle
}

\author{
Ralf Seidl, for the Belle collaboration ${ }^{1}$ \\ RIKEN \\ E-mail: ralf.seidlegmail.com
}

Fragmentation functions provide a substantial tool to understand the formation of confined objects from asymptotically free partons. They can also be used in the study of the spin structure of the nucleon. In particular the access to the flavor information and to quark transversity requires fragmentation function information. The Belle expriment provides a number of measurements to address these. Some of the most recent results include the measurements of hyperon and charmed baryon cross sections and their total production rates. Also the surprising nonzero $\Lambda$ polarization has been recently reported. Last, also the measurement of differential cross sections for di-hadrons as a function of mass and fractional energy have been reported. The related dihadron fragmentation functions are required, together with our previously published interference fragmentation functions to extract transversity from SIDIS and proton-proton collisions.

XXVI International Workshop on Deep-Inelastic Scattering and Related Subjects (DIS2018)

16-20 April 2018

Kobe, Japan

\footnotetext{
${ }^{1}$ Speaker.
} 


\section{Introduction}

Fragmentation functions, FFs, describe the formation of final-state, confined hadrons from asymptotically free partons. Just as the parton distribution functions, PDFs, these are nonperturbative objects which need to be extracted from experiment. However, unlike PDFs, these cannot be accessed from Lattice simulations either. It is more likely that a hadron with matching valence flavor to the initial parton is produced at high momentum fraction $z$ relative to the parton momentum. Therefore, the hadron type relates to the initial parton and it can be used to access flavor in semi-inclusive DIS or hadron-hadron collisions. Additionally, spin dependent fragmentation

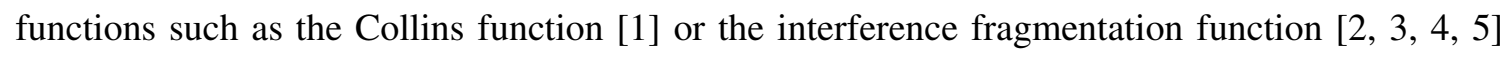
provide access to chiral-odd distributions functions such as quark transversity [ $[$ ] and thus enable measurements which are not possible in inclusive DIS. Additionally, various other fragmentation functions explicitly probe the transverse momentum dependence of the fragmentation process, dihadron fragmentation, heavy flavor fragmentation and polarizing fragmentation. For many of these fragmentation related measurements electron-positron annihilation is the preferred process since the initial state contains no hadrons. The large amount of data accumulated at the Belle experiment at a center of mass energy of $10.58 \mathrm{GeV}$ is ideally suited for these fragmentation function measurements. Several recently published measurements are discussed in the following sections.

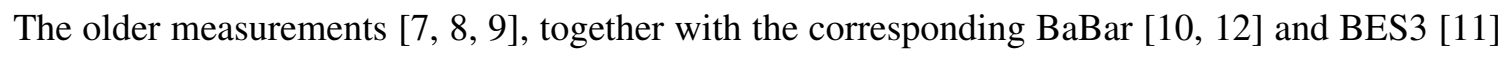
measurements have already successfully been used in global fragmentation fits and in particular

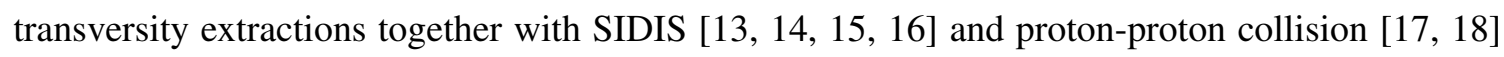
data [107, 201, 21], 22]].

\section{Baryon fragmentation}

The fragmentation of strange and charmed baryons provides information on how fragmentation changes with the mass of the created hadron as well as the mass of the corresponding partons. Furthermore, it also probes information on how the diquarks are produced in the fragmentation process in order to produce baryons from the initial partons. Belle has measured the production cross sections as a function of $x_{p}$ which is the normalized baryon momentum after taking the mass of the particle into account. Various $\Lambda, \Sigma$ and $\Omega$ as well as $\Lambda_{C}$ and $\Sigma_{C}$ cross sections were extracted. The hyperons, as seen in Fig. $\square$ behave very similar to light meson fragmentation functions which peak at low fractional momenta and fall off rapidly. In contrast, charmed baryons, also shown in Fig. I, peak at large $x_{p}$ and the peak position is increasing with baryon mass. This shows that the charmed hadron carries a large fraction of the initial charmed parton. This is again consistent with the behavior seen in charmed meson fragmentation. When integrating the cross sections over the fractional momentum, and extrapolating uncovered areas particularly at low $x_{p}$, one can obtain the total production rates for these hyperons and charmed baryons. When normalized by the hadron's spin degrees of freedom, hyperons appear in a linear relation with the mass and different slopes depending on strangeness. Also charmed baryons follow such a linear behavior but the productions rates are substantially higher than the extrapolated hyperon slopes would suggest. Also, the 

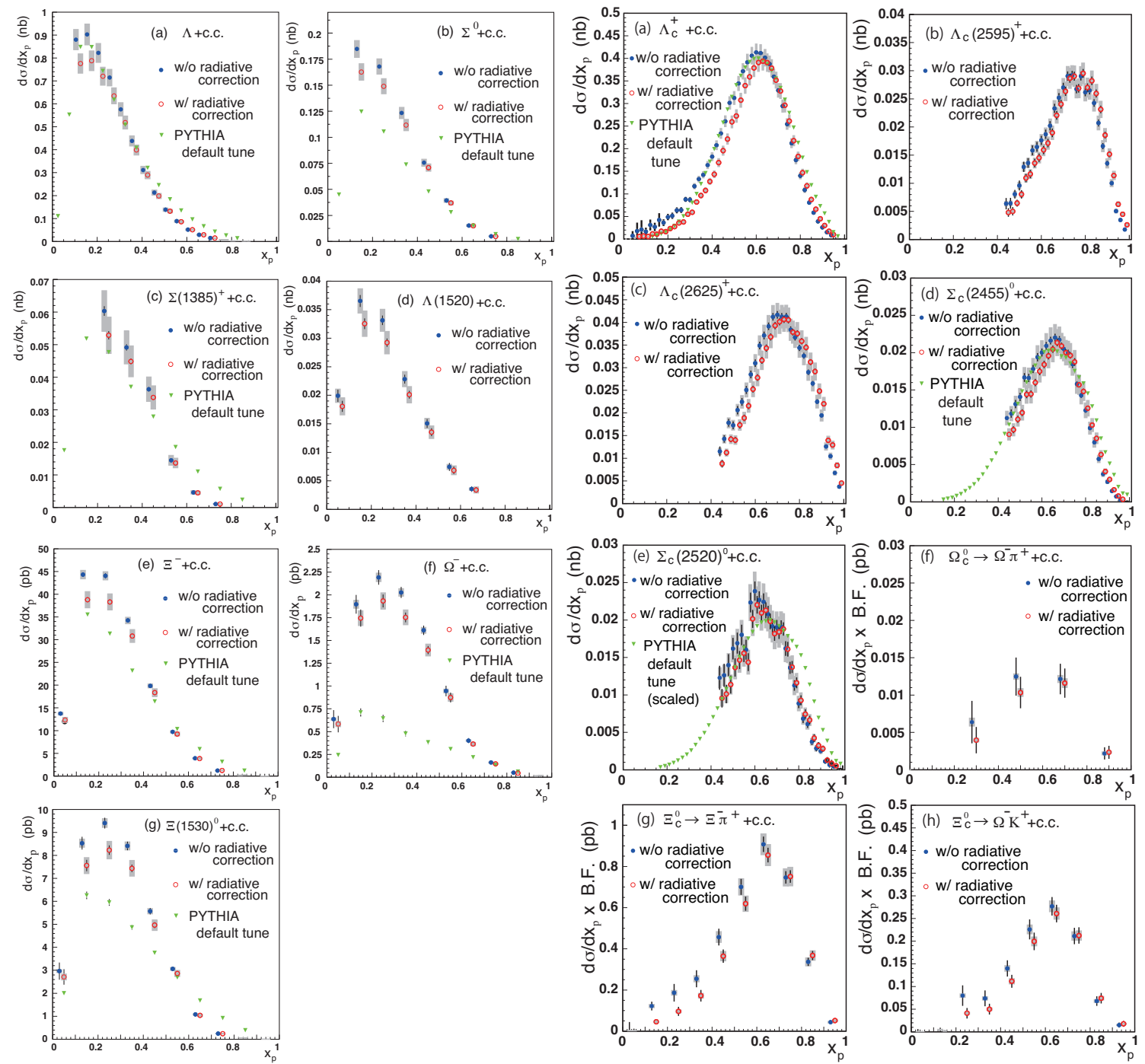

Figure 1: Left: Hyperon cross sections as a function of $x_{p}$ before (blue, full circles) and after (red, open circles) radiative correction. Right: Charmed baryon cross sections as a function of $x_{p}$ before (blue, full circles) and without (red, open circles) radiative correction. The predictions based on the default PYTHIA setting are shown as green triangles.

production rates for $\Lambda_{C}$ baryons is larger than $\Sigma_{C}$ baryons which is consistent with diquark model predictions where the two light quarks can either be in a scalar or vector state.

Another important, preliminary Belle measurement provides the first indication of a polarizing fragmentation function. Here, a nonzero transverse polarization of Lambda baryons is observed for nonzero transverse momentum, see Fig. ㅁ. The polarization is rising with fractional energy but the transverse momentum dependence is nontrivial. To understand these findings better, a charged pion or kaon was required in the opposite hemisphere in order to gain sensitivity to various initial quark-antiquark flavors. It is found that in particular at low fractional energies of the $\Lambda$ this impacts the polarization substantially as even the sign changes between those correlated with a positive and negative pions. According to MC simulations, up and anti-up quark fragmentation into the $\Lambda$ is 
dominating here which suggests different polarizations for these two flavors. At higher fractional energies the strange quark fragmentation plays a major role and the polarizations for both pion charges become comparable. The results are currently being finalized and will be published soon.

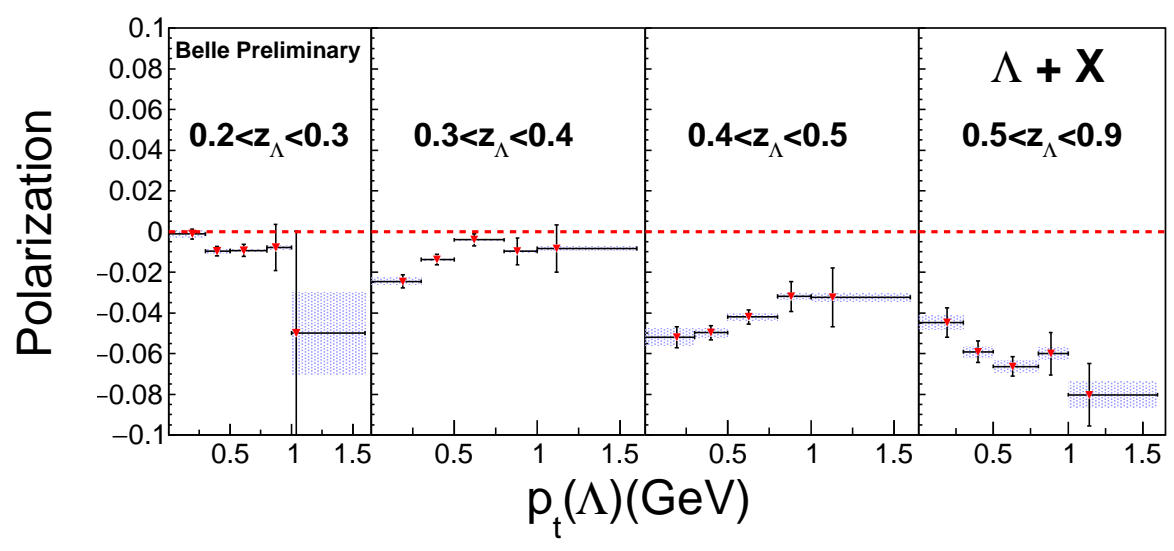

Figure 2: $\Lambda$ polarization (red, full circles) as a function of transverse momentum relative to the thrust axis in bins of $z$.

\section{Di-hadron fragmentation}

Di-hadron fragmentation functions have already been obtained by Belle as a function of the fractional energies of the individual hadrons [25]. For hadrons in opposite hemispheres, as defined by the plane perpendicular to the thrust axis, these are related to the combination of two single hadron fragmentation functions. The different hadron type and charge combinations then provide flavor information otherwise not accessible in electron-positron annihilation. If the two hadrons are detected in the same hemisphere explicit di-hadron fragmentation becomes the relevant process. The assumption of fragmentation of two hadrons from the same parton has been experimentally validated as the sum of the two fractional energies does not exceed unity in the same hemisphere. Di-hadron fragmentation functions as a function of invariant mass and the fractional energy of the hadron pair are essential as the unpolarized baseline for the transversity measurements using the interference fragmentation function in SIDIS and polarized proton-proton collisions. Belle has measured the di-hadron cross sections as a function of invariant mass and fractional energy for every charged pion and kaon combination [26]. In the opposite-sign pairs the corresponding vector meson contributions, $\rho^{0}, K^{0}$ and $\Phi$ are clearly visible as can be seen in Fig. [3 for the pion pairs. The $D^{0}$ meson is also visible in all three charge combinations as are a few other resonances. The same-sign cross sections do generally not show any peaking behavior as they cannot originate directly from resonances. However, in all distributions bumps from hadrons originating from the same decay chain appear, as those are kinematically correlated. 


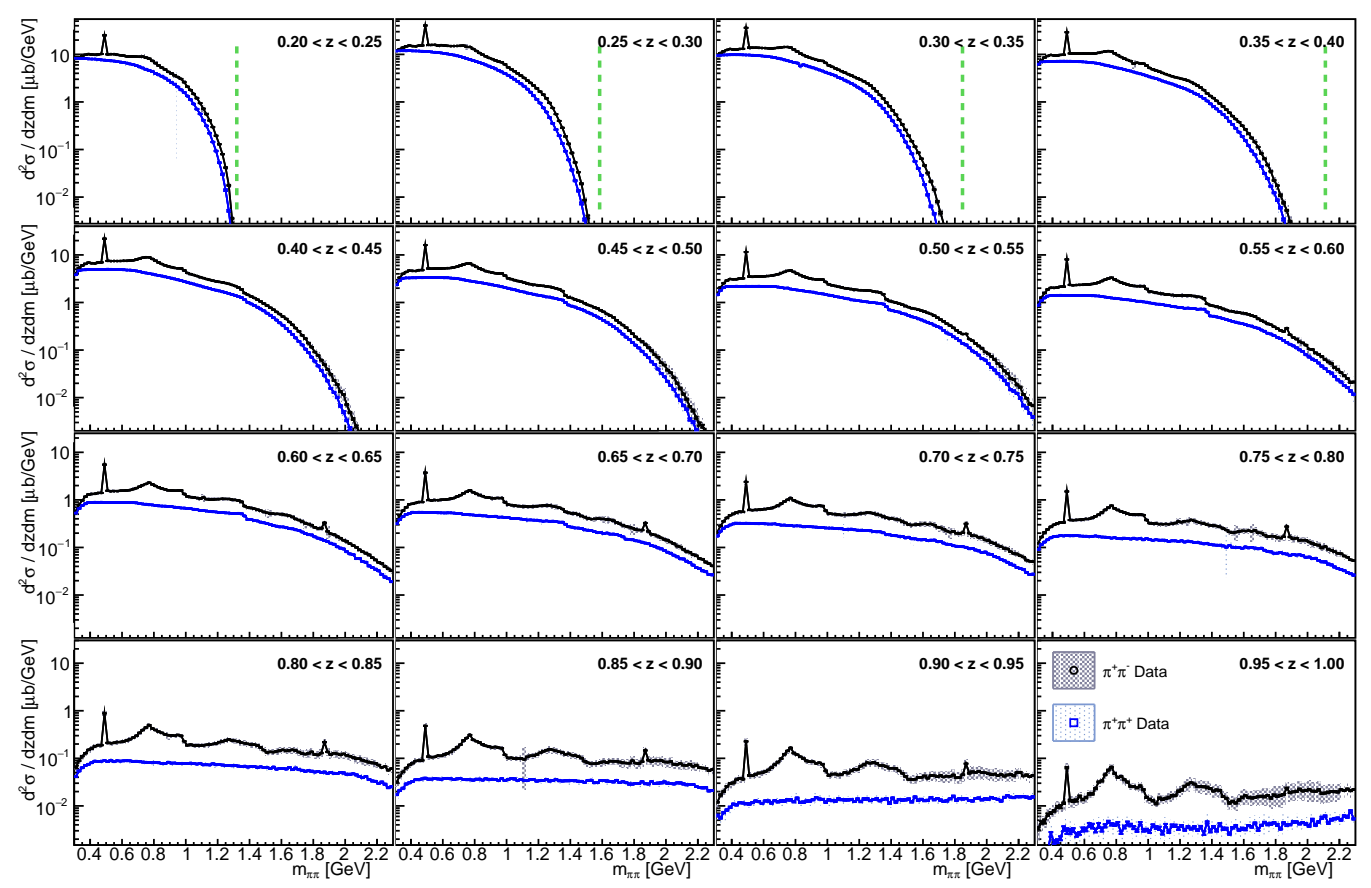

Figure 3: Opposite sign (black circles) and same sign (blue squares) dipion cross sections as a function of invariant mass in bins of $z$.

\section{Ongoing measurements}

While the $\Lambda$ polarization result is being finalized, several other fragmentation related results are currently being prepared. One very large uncertainty in all transverse momentum dependent measurements such as the Sivers and Collins effects in SIDIS is the separation of intrinsic transverse momentum and that produced in the fragmentation process. It is important to study the latter in electron-positron annihilation to significantly reduce these uncertainties. Belle is currently studying two types of transverse momentum dependent fragmentation related observables. One analysis makes use of two hadrons in opposite hemispheres and either calculates the relative transverse momentum of one hadron relative to the other or, theoretically preferable, the transverse momentum of the virtual photon in the system where the two hadrons are back-to-back. This method of accessing transverse momentum dependent fragmentation is straightforward, but involves the convolution of two transverse momenta and fragmentation functions. A slightly cleaner access comes from using a single hadron and calculating the transverse momentum relative to a proxy for the initial parton such as the thrust or jet axis. In this case, apart from the convolution between parton and thrust momentum, one probes a transverse momentum dependent single hadron fragmentation function. Both types of analysis are expected to be finalized soon. Additionally, the analysis of Collins asymmetries including neutral hadrons as well as that including kaons are currently ongoing.

\section{References}

[1] J. C. Collins, Nucl. Phys. B396, 161 (1993)

[2] J. C. Collins, S. F. Heppelmann and G. A. Ladinsky, Nucl. Phys. B420, 565 (1994) 
[3] R. L. Jaffe, X. Jin and J. Tang, Phys. Rev. Lett. 80, 1166 (1998)

[4] M. Radici, R. Jakob and A. Bianconi, Phys. Rev. D 65, 074031 (2002)

[5] D. Boer, R. Jakob and M. Radici, Phys. Rev. D 67, 094003 (2003), D. Boer, Nucl. Phys. B806, 23 (2009)

[6] J. P. Ralston and D. E. Soper, Nucl. Phys. B152, 109 (1979).

[7] R. Seidl et al. (Belle Collaboration), Phys. Rev. Lett. 96, 232002 (2006) R. Seidl et al. (Belle Collaboration), Phys. Rev. D 78, 032011 (2008); Erratum-ibid. D 86, 039905 (2012)

[8] A. Vossen et al. (Belle Collaboration), Phys. Rev. Lett. 107, 072004 (2011)

[9] M. Leitgab et al. [Belle Collaboration], Phys. Rev. Lett. 111, 062002 (2013)

[10] J. P. Lees et al. (BaBar Collaboration), Phys. Rev. D 90, 052003 (2014)

[11] M. Ablikim et al. (BESIII Collaboration), Phys. Rev. Lett. 116, 042001 (2016)

[12] J. P. Lees et al. (BaBar Collaboration), Phys. Rev. D 92, 111101(R) (2015)

[13] A. Airapetian et al. (HERMES Collaboration), Phys. Rev. Lett. 94, 012002 (2005)

[14] C. Adolph et al. (COMPASS Collaboration), Phys. Lett. B 717, 376 (2012)

[15] A. Airapetian et al. (HERMES Collaboration), JHEP 0806, 017 (2008)

[16] C. Adolph et al. (COMPASS Collaboration), Phys. Lett. B 713, 10 (2012) C. Adolph et al. (COMPASS Collaboration), Phys. Lett. B 736, 124 (2014)

[17] J. K. Adkins et al. (STAR Collaboration), Int. J. Mod. Phys. Conf. Ser. 40, 1660040 (2016).

[18] L. Adamczyk et al. (STAR Collaboration), Phys. Rev. Lett. 115, 242501 (2015)

[19] M. Anselmino, et al., Phys. Rev. D 75, 054032 (2007)

[20] Z. B. Kang, A. Prokudin, P. Sun and F. Yuan, Phys. Rev. D 93, 014009 (2016)

[21] M. Anselmino, et al., Phys. Rev. D 93, 034025 (2016)

[22] A. Bacchetta, A. Courtoy and M. Radici, Phys. Rev. Lett. 107, 012001 (2011) A. Bacchetta, A. Courtoy and M. Radici, JHEP 1303, 119 (2013) M. Radici, A. Courtoy, A. Bacchetta and M. Guagnelli, JHEP 1505, 123 (2015)

[23] M. Niiyama et al. [Belle Collaboration], Phys. Rev. D 97, no. 7, 072005 (2018)

[24] A. Abdesselam et al. [Belle Collaboration], arXiv:1611.06648 [hep-ex].

[25] R. Seidl et al. [Belle Collaboration], Phys. Rev. D 92, no. 9, 092007 (2015)

[26] R. Seidl et al. [Belle Collaboration], Phys. Rev. D 96, no. 3, 032005 (2017) 